\title{
A case report of hepatocellular carcinoma and concurrent pancreatic neuroendocrine tumor and renal angiomyolipoma in a patient with tuberous sclerosis
}

\author{
Anjali G. Bal ${ }^{* 1}$, Osama Rahma ${ }^{2}$, Mitchell Rosner ${ }^{3}$ \\ ${ }^{1}$ Department of Medicine, University of Virginia, Charlottesville, Virginia, USA \\ ${ }^{2}$ Department of Medicine, Division of Hematology and Oncology, University of Virginia, Charlottesville, Virginia, USA \\ ${ }^{3}$ Department of Medicine, Division of Nephrology, University of Virginia, Charlottesville, Virginia, USA
}

Received: January 28, 2016

DOI: $10.5430 /$ crim.v3n2p26
Accepted: March 13, 2016

Online Published: March 30, 2016

URL: http://dx.doi.org/10.5430/crim.v3n2p26

\begin{abstract}
Tuberous sclerosis complex (TSC) is an inherited, neurocutaneous disorder that presents with the formation of hamartomas involving multiple organ systems. Most commonly affected sites include the kidney, heart, and brain. Pancreatic and hepatic lesions are less commonly reported, and there are currently no definitive screening guidelines for abdominal neoplasms in tuberous sclerosis patients. In this case report, we present a patient with TSC who developed pancreatic neuroendocrine tumor, renal angiomyolipoma, and hepatocellular carcinoma.
\end{abstract}

Key Words: Tuberous sclerosis, Hepatocellular carcinoma, Pancreatic neuroendocrine tumor, Renal angiomyolipoma

\section{INTRODUCTION}

Tuberous sclerosis complex (TSC) is an autosomal dominant, neurocutaneous disorder that presents with the development of hamartomas throughout multiple organ systems. ${ }^{[1]} \mathrm{Al}-$ though it is generally associated with benign neoplasms, patients with TSC are at increased risk for malignancy. ${ }^{[2]}$ The clinical manifestations of TSC are caused by mutations in the TSC 1 and TSC2 genes. These mutations result in disinhibition of mTORC1, a protein kinase responsible for cell growth and proliferation. Activation of mTORC1 leads to uncontrolled cell growth. In TSC, this commonly presents as renal angiomyolipomas, rhabdomyomas, and central nervous system (CNS) tumors. ${ }^{[3]}$ Less commonly, TSC patients develop primary tumors in the pancreas and liver. ${ }^{[4,5]}$ In this case report, we present a patient with TSC who developed renal angiomyolipoma, hepatocellular carcinoma (HCC) and a pancreatic neuroendocrine tumor.

\section{CASE REPort}

A 28-year-old male with a history of tuberous sclerosis and Lennox-Gastaut syndrome was brought by his family to our clinic for treatment recommendations for HCC and pancreatic neuroendocrine tumor. Three years prior to presenting to our clinic, he had CT imaging of his abdomen and pelvis as part of an evaluation for status epilepticus. Imaging revealed multiple lesions in the liver, bilateral kidneys and

\footnotetext{
${ }^{*}$ Correspondence: Anjali G. Bal; Email: AGB2Z@hscmail.mcc.virginia.edu; Address: Department of Medicine, University of Virginia, Charlottesville, Virginia, USA.
} 
pancreas. The largest liver lesion at that time measured approximately $22 \mathrm{~mm}$. A $2.4 \mathrm{~cm} \times 3.1 \mathrm{~cm}$ enhancing lesion was seen in the right upper kidney pole and a $5 \mathrm{~cm}$ heterogenous enhancing mass was observed in the left kidney. He later underwent endoscopic ultrasound guided FNA of the pancreatic tail lesion and one of the hepatic lesions. The pancreatic lesion histopathology was consistent with pancreatic endocrine neoplasm. The liver FNA was felt to be an insufficient sample. One group of cells had features suggestive of neuroendocrine tumor, but most of the samples were reactive hepatocytes. When the sample was reviewed at a second institution, the sample size was felt to be inadequate; however there was strong suspicion for a hepatic neoplasm as opposed to neuroendocrine metastasis. A second liver sample was obtained, this time a core biopsy. These cells were strongly positive for HepParl, suggesting the lesion was hepatic in origin and not metastatic neuroendocrine tumor. Polyclonal CEA demonstrated a canicular pattern, suggestive of HCC. The final histopathology result was confirmed to be well differentiated hepatocellular carcinoma. He also underwent renal biopsy that was HMB-45 positive and consistent with angiomyolipoma. He had a repeat CT scan about 5 months after his initial biopsies that showed stable pancreatic lesions, one in the uncinate measuring $21 \mathrm{~mm} \times 16 \mathrm{~mm}$ and one in the tail of the pancreas measuring $9 \mathrm{~mm} \times 9 \mathrm{~mm}$. His liver lesions appeared stable, with the largest lesion in segment $\mathrm{V}$ measure $5.3 \mathrm{~cm} \times 3.5 \mathrm{~cm}$.

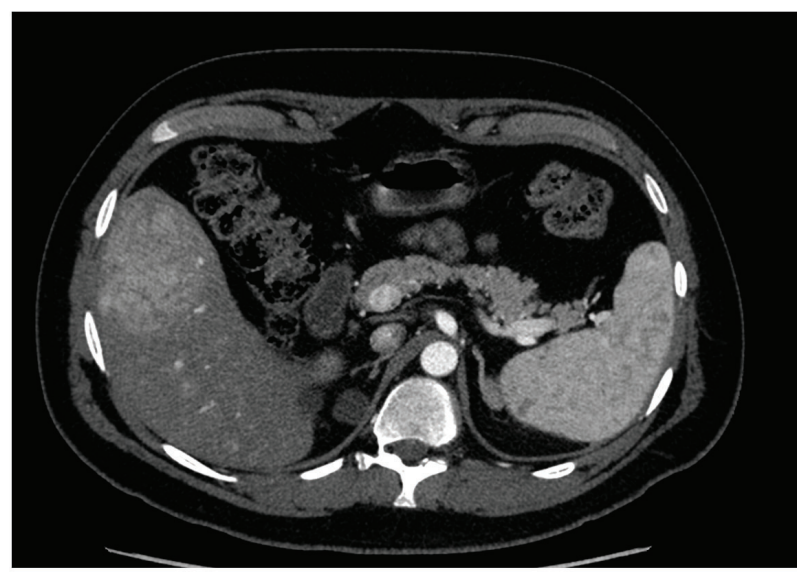

Figure 1. CT imaging demonstrating large arterial enhancing hepatic lesion

Given the patient's functional status (he is bed bound), the family felt that conservative management for his well differentiated HCC and neuroendocrine tumor with serial cross sectional imaging would be more in line with the patient's and family's goals of care. Although he had poor functional status, his family still wished to pursue imaging as part of his ongoing care in addition to treatment with everolimus for

Published by Sciedu Press renal angiomyolipoma. We continued serial abdominal cross sectional imaging about every three months. Approximately 8 months following his initial visit, CT imaging showed multiple new poorly marginated arterial phase enhancing hepatic lesions. These appeared to be of the same origin, suggestive of multifocal HCC. Also noted was interval increase in size of the hepatic lesion in segment $\mathrm{V}$ as seen in Figure 1.

We discussed obtaining biopsies of the new lesions. However, given the patient's poor functional status and overall indolent nature of well differentiated HCC and neuroendocrine tumors, the decision was made to continue active surveillance with repeat imaging and to continue treatment with everolimus for renal angiomyolipoma. On repeat imaging about 3 months later, the hepatic lesions appeared stable with some having decreased in size. His pancreatic lesion in the uncinated process (see Figure 2) and tail as well as renal lesions appeared stable, with interval decrease in the left renal mass.

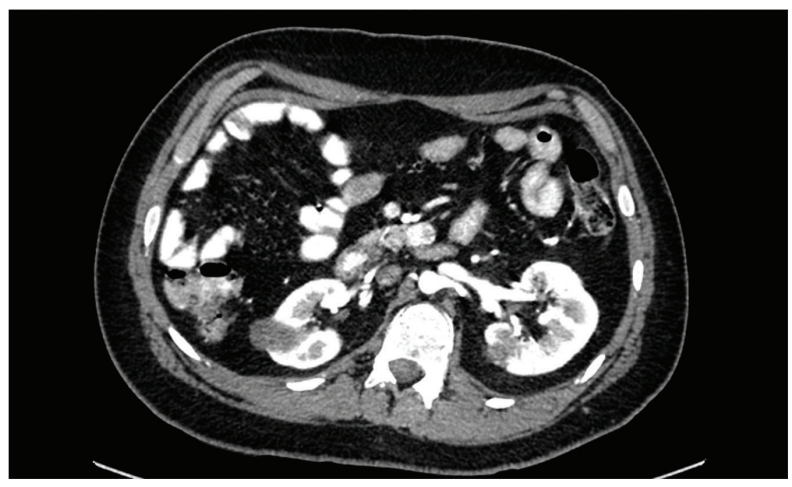

Figure 2. CT imaging demonstrating $15 \mathrm{~mm}$ hyperintense lesion in the uncinated process

\section{Discussion}

The case presented above illustrates two neoplasms that are not commonly associated with tuberous sclerosis complex appearing concurrently. While pancreatic neuroendocrine tumors in TSC have been reported in multiple case reports, ${ }^{[4]}$ hepatocellular carcinoma is far less common and was only found in one case report per our literature search. ${ }^{[5]}$

Neoplasms are a common manifestation of TSC. While benign neoplasms are far more common, patients with TSC are at increased risk for malignant lesions. Common tumors include cardiac rhabdomyomas, subendyomas, and renal angiomyolipomas. ${ }^{[2]}$ Many of these develop in childhood, but renal angiomyolipomas more commonly present in adults. In the literature, it is reported that about $70 \%-90 \%$ of adult TSC patients develop bilateral renal angiomyolipomas. ${ }^{[6]}$

While not as commonly reported as renal anigomyolipomas and rhabdomyomas, neuroendocrine tumors affecting the 
pancreas have also been reported in TSC. Pancreatic neuroendocrine tumors (PanNETs) generally originate from mature endocrine cells in the pancreas and can be divided into two broad categories, functional or non-functional. ${ }^{[7]}$ The WHO classifies panNETs into three categories: well differentiated endocrine tumor, as observed in our patient, has an indolent clinical course, moderately-differentiated endocrine carcinoma, and poorly differentiated endocrine carcinoma which is the most aggressive. ${ }^{[8]}$ Pancreatic NETs can occur spontaneously, or they can be associated with familial syndromes like MEN-1 and TSC where they more commonly occur in early adulthood. ${ }^{[9,10]}$ The development of PanNETs may be attributed to disinhibition of mTOR. This may explain the stable disease our patient had on everolimus, an mTOR inhibitor now approved for use on pancreatic NETs. ${ }^{[7]} \mathrm{Hep}-$ atic involvement in TSC has most commonly been reported in the form of hepatic angiomyolipoma. ${ }^{[6]}$ Hepatocellular carcinoma is much less common, and our literature search yielded one case report that demonstrated HCC and hepatic angiomyolipoma occurring concurrently in a TSC patient. ${ }^{[5]}$ While angiomyolipoma is a benign neoplasm, hepatocellular carcinoma represents a more aggressive type of liver tumor. $^{[11]}$
TSC2 dysfunction has been implicated in the pathogenesis of both HCC and pancreatic NETs. Decreased TSC2 expression was observed in about $35 \%$ of panNETs. ${ }^{[12]}$ Additionally, a recent study found that loss of TSC2 function was common in HCC. That same study showed that a loss of TSC2 function also predicted a better response to everolimus, an mTOR inhibitor our patient is receiving for renal angiomyolipoma treatment. ${ }^{[11]}$

The findings in our patient raise questions about screening and prognosis for patients with TSC. Currently, there are no definitive screening guidelines for abdominal imaging in patients with TSC. Given the complexity and variability with which TSC can present, screening for gastrointestinal manifestations is tailored for individual patients. ${ }^{[13]}$ To our knowledge, this is the first case reported of pancreatic NET, HCC, and renal angiomyolipoma occurring concurrently in a patient with TSC. More research and investigation is needed to determine if these three neoplasms occurring together affects treatment choices or prognosis.

\section{CONFlicts OF INTEREST Disclosure}

The authors have declared no conflicts of interest.

\section{REFERENCES}

[1] Lenci I, Angelico M, Tisone G, et al. Massive hepatic angiomyolipoma in a young woman with tuberous sclerosis complex: Significant clinical improvement during tamoxifen treatment. J Hepatol. 2008; 48: 1026-1029. http://dx.doi.org/10.1016/j.jhe p. 2008.01 .036

[2] Al-Saleem T, Wessner L, Scheithauer B, et al. Malignant tumors of the kidney, brain, and soft tissues in children and young adults with the tuberous sclerosis complex. Cancer. 1998; 83(10): 22082216. http://dx.doi.org/10.1002/(SICI) 1097-0142(1998 1115) $83: 10<2208:$ : AID-CNCR21>3.0.CO; $2-\mathrm{K}$

[3] DiMario Jr FJ, Sahin M, Ebrahimi-Fakhari D. Tuberous Sclerosis Complex. Pediatr Clin North Am. 2015; 62: 633-648. http: //dx.doi.org/10.1016/j.pcl.2015.03.005

[4] Larson AM, Hedgire SS, Deshpande V. Pancreatic neuroendocrine tumors in patients with tuberous sclerosis complex. Clin Genet. 2012; 82: 558-563. http://dx.doi.org/10.1111.j.1399-0004.20 $11.01805 . \mathrm{x}$

[5] Yang B, Chen WH, Shi PZ, et al. Coincidence of hepatocellular carcinoma and hepatic angiomyolipomas in tuberous sclerosis complex: a case report. World J Gastroenterol. 2008; 14(5): 812-814 http://dx.doi.org/10.3748/wjg.14.812

[6] Curatolo P, Bombardieri R, Jozwiak S. Tuberous Sclerosis. The Lancet. 2008; 372: 657-68. http://dx.doi.org/10.1016/S01 40-6736 (08) 61279-9

[7] Zhou C, Zhang J, Zheng Y, et al. Pancreatic neuroendocrine tumors: a comprehensive review. Int J Cancer. 2012; 131: 1013-1022. http://dx.doi.org/10.1002/ijc. 27543
[8] Kloppel G, Perren A, Heitz PU. The gastroenteropancreatic neuroendocrine cell system and its tumors: the WHO classification. Ann N Y Acad Sci. 2004; 1014: 13-27. PMid:15153416 http: //dx.doi.org/10.1196/annals.1294.002

[9] Lodish MB, Stratakis CA. Endocrine tumors in neurofibromatosis type 1, tuberous sclerosis, and related syndromes. Best Pract Res Clinical Endocrinol Metab. 2010; 24: 439-449. http://dx.doi.o $\mathrm{rg} / 10.1016 / \mathrm{j}$. beem. 2010.02.002

[10] Dworakowska D, Grossman AB. Are neuroendocrine tumors a feature of tuberous sclerosis? A systematic review. Endocrine-Related Cancer. 2009; 16: 45-58. http://dx.doi .org/10.1677/ERC-0 9-0142

[11] Huynh H, Hao HX, Chan SL, et al. Loss of tuberous sclerosis complex 2 (TSC2) is frequent in hepatocellular carcinoma and predicts response to mTORC1 inhibitor everolimus. Mol Cancer Ther. 2015; 14(5): 1224-1235. http://dx.doi .org/10.1158/1535-7 163. MCT-14-0768

[12] Missiaglia E, Dalai I, Barbi S, et al. Pancreatic endocrine tumors: expression profiling evidences a role for AKT- mTOR pathway. Journal of Clinical Oncology. 2010; 28(2): 245-255. http://dx.doi.org $/ 10.1200 /$ JCD .2008 .21 .5988

[13] Krueger DA, Northup H. Tuberous sclerosis complex surveillance and management: recommendations of the 2012 international tuberous sclerosis complex consensus conference. Pediatr Neurol. 2013; 49: 255-265. PMid:24053983 http://dx.doi.org/10.1016/j .pediatrneurol.2013.08.002 